TITLE:

\title{
The application of robustness analysis to the conflict with incomplete information
}

$\operatorname{AUTHOR}(\mathrm{S})$ :

Sakakibara, H; Okada, N; Nakase, D

\section{CITATION:}

Sakakibara, $\mathrm{H}$...[et al]. The application of robustness analysis to the conflict with incomplete information. IEEE TRANSACTIONS ON SYSTEMS MAN AND CYBERNETICS PART C-APPLICATIONS AND REVIEWS 2002, 32(1): 14-23

ISSUE DATE:

2002-02

URL:

http://hdl.handle.net/2433/40009

\section{RIGHT:}

(c)2002 IEEE. Personal use of this material is permitted. However, permission to reprint/republish this material for advertising or promotional purposes or for creating new collective works for resale or redistribution to servers or lists, or to reuse any copyrighted component of this work in other works must be obtained from the IEEE. 


\title{
The Application of Robustness Analysis to the Conflict With Incomplete Information
}

\author{
Hiroyuki Sakakibara, Member, IEEE, Norio Okada, and Daisuke Nakase
}

\begin{abstract}
When players with different interests try to achieve a better state, conflicts among players arise. Conflicts may arise also among public players. For example, a local government may insist on the interest of the region while the national government represents the interests of the whole country.

Conflict analysis is one of the methods to model such conflicts mathematically. Its stability analysis specifies stable states based on the ordinal information on players' preferences. However, if the preference of a player is private, stability of states is not known. In such a case, players or third parties have to collect additional information on other players' preference. It is necessary to specify the minimum information to collect.

In this paper, graph model for conflict resolution (GMCR) is extended for the cases with incomplete information. Then, the generalized robustness analysis is proposed to specify the minimum conditions for stability of states. Finally, robustness analysis is applied to the conflict on water resources development.
\end{abstract}

Index Terms-Conflict with incomplete information, graph model for conflict resolution (GMCR), robustness analysis, two-player conflict.

\section{INTRODUCTION}

$\mathbf{F}$ RASER and Hipel [1] proposed conflict analysis based on metagame theory [2]. Conflict analysis defines stability of states and specifies stable states. Nash equilibrium in game theory is one of the concepts of stability. Fang et al. [3] extended the methodology and proposed graph model for conflict resolution (GMCR). In GMCR, agents' moves between states are extended to include common and irreversible moves.

In order to analyze the stability of states, it is critical to know the preferences of players. However, it is often difficult to obtain complete information. That is why minimum conditions for stability should be specified before the inspection of preferences. Okada et al. [4] proposed robustness analysis to identify the minimum conditions on players' preferences in the 2-player conflicts where one player's preference is not known to another player. On the other hand, the third party may not have enough information on both players' preferences.

In this paper, robustness analysis is extended to apply to the graph model for 2-player conflicts where both players' prefer-

Manuscript received December 10, 1999; revised November 13, 2001 and December 20, 2001. This paper was recommended by Associate Editor L. Fang.

H. Sakakibara is with the Department of Civil Engineering, Yamaguchi University, Yamaguchi 755-8611, Japan (e-mail: hsakaki@jim2.civil.yamaguchiu.ac.jp).

N. Okada is with the Disaster Prevention Research Institute, Kyoto University, Kyoto 611-0011, Japan (e-mail: okada@imdr.dpri.kyoto-u.ac.jp).

D. Nakase is with the Obayashi Corporation, Osaka 540-8584, Japan (e-mail: dnakase@o-net.obayashi.co.jp).

Publisher Item Identifier S 1094-6977(02)04678-3.

\begin{tabular}{|c|c|c|}
\hline Player 1 & Confess & Not Confess \\
\hline Confess & 1 & 2 \\
\hline Not Confess & 3 & 4 \\
\hline
\end{tabular}

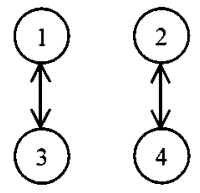

Player 1's feasible transition $\left(D_{1}\right)$

$P_{1}(1)=2, P_{1}(2)=4, P_{1}(3)=1, P_{1}(4)=3$

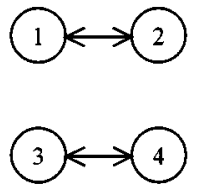

Player 2's feasible transition $\left(\mathrm{D}_{2}\right)$

$P_{2}(1)=2, P_{2}(2)=1, P_{2}(3)=4, P_{2}(4)=3$
Fig. 1. Strategic form (upper) and graph form (below) of "prisoners' dilemma."

ences are not known each other. In Section II, GMCR is extended for the cases with incomplete preference information. In Section III, generalized robustness analysis is proposed. In Section IV, the methodology of robustness analysis is applied to the conflict on water resources development.

\section{GRAPH MODEL FOR CONFLICT RESOLUTION WITH INCOMPLETE INFORMATION}

\section{A. Graph Model for Conflict Resolution (GMCR)}

Fang et al. [3] proposed the GMCR as the representation of conflicts among players. Let $N=\{1,2, \ldots, n\}$ be the set of players and $K=\{1,2, \ldots k\}$ be the set of states of the conflict. We also define $N$-tuple $\left\{D_{i}\right\}(i=1,2, \ldots, n)$ as the set of directed graphs that $D_{i}=\left(K, V_{i}\right)$. The set of arcs $V_{i}$ means player $i^{\prime}$ s possible move between states. Let $k_{1} k_{2}$ be the arc from the state $k_{1}$ to the state $k_{2}$. If $k_{1} k_{2} \in V_{i}$, player $i$ can move from the state $k_{1}$ to the state $k_{2}$ unilaterally. We also need to define the payoff function $P_{i}: K \rightarrow R$ ( $R$ : the set of real numbers). Payoff function determines players' evaluations of the state in $K$, and specifies players' preference orders. If $P_{i}\left(k_{1}\right)$ is larger than $P_{i}\left(k_{2}\right)$, player $i$ prefers the state $k_{1}$ to the state $k_{2}$. GMCR is represented by 4-tuple $\{N, K, V, P\}$, where $N=$ $\{1,2, \ldots n\}, K=\{1,2, \ldots k\}, V=\left\{V_{1}, V_{2}, \ldots, V_{n}\right\}$, and $P=\left\{P_{i} \mid K \rightarrow R, i \in N\right\}$. Fig. 1 shows both strategic form and graph form of the "prisoner's dilemma." In this case, $P_{1}(1)=2, P_{1}(2)=4, P_{1}(3)=1, P_{1}(4)=3$ and $P_{2}(1)=$ 2, $P_{2}(2)=1, P_{2}(3)=4, P_{2}(4)=3$, respectively.

The following are other definitions used in GMCR. 


\begin{tabular}{|c|c|c|}
\hline Player 1 & $\begin{array}{c}\text { Not } \\
\text { Cooperate }\end{array}$ & Cooperate \\
\hline $\begin{array}{c}\text { Not } \\
\text { Cooperate }\end{array}$ & 1 & 2 \\
\hline Cooperate & 3 & 4 \\
\hline
\end{tabular}

(The numbers in the matrix mean states.)

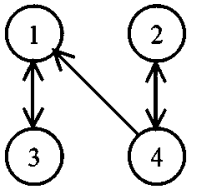

Player 1's feasible transition $\left(\mathrm{D}_{1}\right)$

$P_{1}(1)=2, P_{1}(2)=4, P_{1}(3)=1, P_{1}(4)=3$

Player 2's feasible transition $\left(\mathrm{D}_{2}\right)$

$P_{2}(1)=2, P_{2}(2)=1, P_{2}(3)=4, P_{2}(4)=3$

Fig. 2. Strategic form (upper) and graph form (below) of formulation of cooperation.

a) State $k^{\prime}$ s reachable list $S_{i}(k)$

$k_{1} \in S_{i}(k)$ if player $i$ can move unilaterally from the state $k$ to the state $k_{1}\left(k k_{1} \in V_{i}\right)$.

b) State $k^{\prime}$ s unilateral improvement (UI) $S_{i}^{+}(k)$

$k_{1} \in S_{i}^{+}(k)$ if $k_{1} \in S_{i}(k)$ and $P_{i}\left(k_{1}\right)>P_{i}(k)$.

Compared with strategic form, the major advantage of graph form is that it can represent common and irreversible moves. The definitions of common and irreversible moves are as follows.

Definition:

- The move $k_{1} k_{2}$ is common move if $k_{1} k_{2} \in V_{i}$ and $k_{1} k_{2} \in$ $V_{j}$ for $i, j \in N$ and $i \neq j$.

- The move $k_{1} k_{2}$ is irreversible move if $k_{1} k_{2} \in V_{i}$ and $k_{2} k_{1} \notin V_{i}$ for $i \in N$.

In strategic form, states are defined as the combination of players' strategies. As a result, the move between two states is possible only when one player changes its strategy. However, in real conflicts, plural players may be able to move to the same states, and the choice such as "nuclear attack" in military conflicts, leads a conflict to the irreversible result, which cannot be returned. GMCR can incorporate common and irreversible moves into the model.

Fig. 2 shows the game on the formulation of cooperation between two players. The difference from prisoner's dilemma in Fig. 1 is the existence of common and irreversible moves from the state 4 to the state 1 . It is assumed that both players have two alternatives, "collaborate" and "not collaborate" in strategic form. To realize cooperation, both players need to take the alternative "collaborate." However, cooperation collapses even if one player changes its alternative from "collaborate" to "not collaborate." Once cooperation is collapsed by the common move, players cannot return the same path to cooperative state directly. They have to change their strategies one by one again.

\section{B. Definition of Stability and Equilibrium in Two-Player Conflict}

Based on Fang et al. [3], some definitions on stability are shown as follows. Here we assume a 2-player game. The fol- lowing are the solution concepts used in graph model for conflict [3].

\section{Definition:}

- Nash Stability

The state $k$ is Nash stable for player $i$ if, and only if, $i$ cannot improve its payoff by changing his own strategies. In other words, $S_{i}^{+}(k)=\{\varnothing\}$.

- Sequential Stability [4]

The state $k$ is sequentially stable for player $i$ if, and only if, for every $k_{1} \in S_{i}^{+}(k)$, there exists $k_{2} \in S_{j}^{+}\left(k_{1}\right)$ with $P_{i}(k) \geq P_{i}\left(k_{2}\right)$. (Here, $k_{2}$ is called the sanction for player $i$ S UI, $k_{1}$.)

- General Metarationality (GMR)

The state $k$ is general metarational for player $i$ if, and only if, for every $k_{1} \in S_{i}^{+}(k)$, there exists $k_{2} \in S_{j}\left(k_{1}\right)$ with $P_{i}(k) \geq P_{i}\left(k_{2}\right)$.

We use these solution concepts also in the following robustness analysis.

\section{Extension of Graph Model for Conflict Resolution for the Case With Incomplete Preference Information}

Potential users of GMCR are players themselves, consultants advising players, third parties analyzing conflicts, mediator, etc. (Fang et al. [3]). Let us call such users "analysts." In some cases, an analyst does not have complete information on players' preferences. When one player is an analyst, it may not know about its counterparts' preferences. Consultants, third parties, or mediator may have only limited information on all players' preferences.

Before presenting the GMCR with incomplete information, we introduce a binary description for representing ordinal preference. $k_{1} \succ_{i} k_{2}$ means that player $i$ (strictly) prefers $k_{1}$ to $k_{2}$, and $k_{1} \succeq_{i} k_{2}$ means that player $i$ strictly or equally prefers $k_{1}$ to $k_{2}$. On the other hand, $k_{1} \sim_{i} k_{2}$ means that $k_{1}$ and $k_{2}$ are indifferent for player $i$.

Let the pattern of players' preference orders and the set of patterns be $\omega$ and $\Omega$, respectively. When $k$ states are strictly ordered (there are no indifferent states), the number of each player's preference orders amounts to $k$ !. Consequently, the number of patterns of $n$ players' preference orders becomes $(k !)^{n}$ and $|\Omega|=(k !)^{n}$.

If an analyst has complete information, it can recognize $\omega$ exactly. However, if an analyst has only limited knowledge, it only recognizes that the pattern of preference orders is included in the subset of $\Omega$. Let $\pi$ be the subset of $\Omega$ representing an analyst's knowledge on players' preferences. Although an analyst knows that the true pattern of preference orders is certainly included in the set $\pi$, it does not know which is a true pattern in $\pi$.

Using information set $\pi$, we propose GMCR with incomplete information. GMCR under analyst's information set $\pi$ is represented by 4-tuple $\{N, K, V, \pi\}$, where $N=\{1,2, \ldots n\}$, $K=\{1,2, \ldots k\}, V=\left\{V_{1}, V_{2}, \ldots, V_{n}\right\}$, and $\pi \subseteq \Omega$. When $\pi$ is a singleton set, an analyst has complete information.

Here we define the sets that represent analyst's knowledge on preferences. Ordered sets on analyst's information set $\pi$

$\left[\Phi_{i}^{+}(k, \pi), \Phi_{i}^{-}(k, \pi), \Phi_{i}^{\wedge}(k, \pi)\right.$, and $\left.\Phi_{i}^{*}(k, \pi)\right]$ : 
- The state $k_{1}$ belongs to $\Phi_{i}^{+}(k, \pi)$ if $k_{1}$ is preferred to $k$ by player $i$ at every pattern of preference orders in information set $\pi\left(k_{1} \succ_{i} k \forall \omega \in \pi\right)$.

- The state $k_{1}$ belongs to $\Phi_{i}^{\wedge}(k, \pi)$ if $k_{1}$ is equally preferred to $k$ by player $i$ at every pattern of preference orders in information set $\pi\left(k_{1} \sim_{i} k \forall \omega \in \pi\right)$.

- The state $k_{1}$ belongs to $\Phi_{i}^{-}(k, \pi)$ if $k_{1}$ is less preferred to $k$ by player $i$ at every pattern of preference orders in information set $\pi\left(k_{1} \prec_{i} k \forall \omega \in \pi\right)$.

- The state $k_{1}$ belongs to $\Phi_{i}^{*}(k, \pi)$ if $k_{1}$ does not belong to either $\Phi_{i}^{+}(k, \pi), \Phi_{i}^{\wedge}(k, \pi)$, or $\Phi_{i}^{-}(k, \pi)$. In other words, an analyst does not know if player $i$ prefers $k_{1}$ to $k$ or not with information set $\pi$.

The product of reachable list and ordered set is defined as "ordered reachable list." That is

$$
\begin{gathered}
S_{i}^{+}(k, \pi)=S_{i}(k) \cap \Phi_{i}^{+}(k, \pi) \\
S_{i}^{\wedge}(k, \pi)=S_{i}(k) \cap \Phi_{i}^{\wedge}(k, \pi) \\
S_{i}^{-}(k, \pi)=S_{i}(k) \cap \Phi_{i}^{-}(k, \pi) \\
S_{i}^{*}(k, \pi)=S_{i}(k) \cap \Phi_{i}^{*}(k, \pi) .
\end{gathered}
$$

When $\pi$ is a singleton set, $S_{i}^{+}(k, \pi)=S_{i}^{+}(k)$ and $S_{i}^{*}(k, \pi)=\{\varnothing\}$.

\section{Stability in GMCR With Incomplete Information}

Even if the information on players' preferences is incomplete for the analyst, stability analysis for some states can be carried out. For 2-player conflict, definitions for Nash stability, sequential stability, and GMR based on analyst's knowledge can be shown as follows.

Nash Stability Based on Analyst's Knowledge: The state $k$ is Nash stable for player $i$ based on analyst's information set if, and only if, the following condition is satisfied:

$$
S_{i}^{+}(k, \pi) \cap S_{i}^{*}(k, \pi)=\{\varnothing\} .
$$

Sequential Stability Based on Analyst's Knowledge: The state $k$ is sequentially stable for player $i$ based on analyst's knowledge if, and only if, the following condition is satisfied:

$$
\begin{aligned}
k_{1} & \in \Phi_{i}^{-}(k, \pi) d \text { or } S_{j}^{+}\left(k_{1}, \pi\right) \cap\left\{\Phi_{i}^{-}(k, \pi) \cup \Phi_{i}^{\wedge}(k, \pi)\right\} \\
& \neq\{\varnothing\} \quad \forall k_{1} \in S_{i}(k) .
\end{aligned}
$$

General Metarationality Based on Analyst's Knowledge: The state $k$ is general metarational for player $i$ based on analyst's knowledge if the following condition is satisfied:

$$
\begin{aligned}
k_{1} & \in \Phi_{i}^{-}(k, \pi) \text { or } S_{j}\left(k_{1}, \pi\right) \cap\left\{\Phi_{i}^{-}(k, \pi) \cup \Phi_{i}^{\wedge}(k, \pi)\right\} \\
& \neq\{\varnothing\} \quad \forall k_{1} \in S_{i}(k) .
\end{aligned}
$$

Nash stability is decided without any information on other player's preference. If a player does not have any UIs, the state is Nash stable for the player. On the other hand, sequential stability and GMR depends on the player's knowledge on its counterpart's preference. If a player knows that its counterpart has a UI (in sequential stability) that reduces its payoff, the player gives up moving from the current state.
In the next section, robustness analysis that specifies the necessary and sufficient conditions for the stability of states is shown.

\section{GENERALIZATION OF Robustness AnALYsis}

\section{A. Robustness Analysis}

Stability analysis in conflict analysis decides if the corresponding state is stable or not, based on the solution concepts. However, if an analyst does not know players' preferences, it cannot judge the stability of the state.

In the actual situation, an analyst does not necessarily have the complete information at the beginning of the analysis. Therefore, when an analyst needs to know about the stability, it has to collect information and renew its knowledge. Okada et al. [4] proposed robustness analysis to specify the minimum conditions that is necessary to judge the stability of the corresponding state. robustness analysis is a kind of an inverse problem of stability analysis.

Okada et al. [4] proposed robustness analysis for 2-player conflict in which the preference of one player is not known. In this paper, we generalize the methodology to apply 2-player conflict under arbitrary information set of an analyst. The generalized robustness analysis can be applied to the conflict where both players' preferences are not known. Analysts can use the result of robustness analysis as follows.

Players: Although a player knows its own preference, it may not have enough information on its counterpart's preference. Robustness analysis can provide the minimum information guaranteeing that a state can become a resolution of a conflict.

Mediator: Mediator tries to find the state that can be accepted as a compromise by both players. The stable state has high possibility to be accepted by players. However, in many cases, mediator has incomplete information on both players' preferences. If a mediator can confirm the condition for stability that is shown by robustness analysis, it can present the state as a proposal for agreement with conviction.

In the following parts, robustness analysis is generalized.

\section{B. The Conditions for Stability in 2-Player Conflicts}

Here we show the conditions that are necessary for the third party to judge if the corresponding state is stable or not. Since these conditions are specified based on the third party's knowledge, the information sets on the third party's knowledge are used. The sufficient condition for stability of a state is represented by some inequalities on players' preference. We call the set of these conditions "condition set." In the following subsection, conditions for Nash stability, sequential stability, and GMR are formulated.

1) The Condition for Nash Stability: The state $k$ is Nash stable for player $i$ if the UI from state $k$ by player $i$ is empty set $\left[S_{i}^{+}(k)=\{\varnothing\}\right]$. In GMCR under the information set $\pi$, $S_{i}^{+}(k, \pi)$ is the subset of $S_{i}^{+}(k)$, and $S_{i}^{+}(k)$ is included in $S_{i}^{+}(k, \pi) \cup S_{i}^{*}(k, \pi)$ (see Fig. 3). Consequently, the condition that the state $k$ is Nash stable for player $i$ is shown as follows.

(Presumption) $S_{i}^{+}(k, \pi)=\{\varnothing\}$ : For every $k_{1} \in S_{i}^{*}(k, \pi)$

$$
k \succ_{i} k_{1} \text {. }
$$




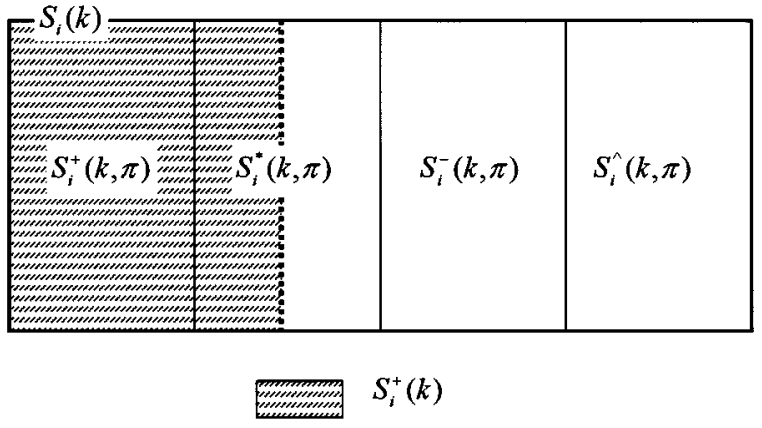

Fig. 3. Relationship between sets.

The number of conditions included in a sufficient condition set becomes $\left|S_{i}^{*}(k, \pi)\right|$. The number of sufficient condition sets for player $i$ is one.

2) The Condition for Sequential Stability: The stake $k$ is sequentially stable for player $i$ if sanction exists for every state included in $S_{i}^{+}(k)$. Since $S_{i}^{+}(k, \pi)$ is the subset of $S_{i}^{+}(k)$, the existence of sanction for player $i^{\prime}$ s movement to the state in $S_{i}^{+}(k, \pi)$ is necessary to guarantee sequential stability of the stake $k$. That is

- For every $k_{1} \in S_{i}^{+}(k, \pi)$

(1) $\quad k_{2} \succ_{j} k_{1}$ and $k \succeq_{i} k_{2}$

$$
\exists k_{2} \in S_{j}^{*}\left(k_{1}, \pi\right)
$$

(2) $k \succeq_{i} k_{2}$

$$
\exists k_{2} \in S_{j}^{+}\left(k_{1}, \pi\right) .
$$

Here, $j=2$ if $i=1$, and $j=1$ if $i=2$. (1) or (2) is the condition that player $i$ does not move to the state which is known as UI for player $i$.

For the state in $S_{i}^{*}(k, \pi)$, it is not known if a state is included in $S_{i}^{+}(k)$ or not. If an analyst obtains information that shows that player $i$ prefers $k$ to the state in $S_{i}^{*}(k, \pi)$, it needs to find information showing existence of sanction. The flowchart for specifying conditions is shown in Fig. 4 and in the following inequalities.

- For every $k_{1} \in S_{i}^{*}(k, \pi) \succ_{i} k_{1}$

(4) $k_{2} \succ_{j} k_{1}$ and $k \succeq_{i} k_{2}$

$$
\exists k_{2} \in S_{j}^{*}\left(k_{1}, \pi\right)
$$

(5) $k \succeq_{i} k_{2}$

$$
\exists k_{2} \in S_{j}^{+}\left(k_{1}, \pi\right) .
$$

(3) is the condition that $k_{1}$ is not UI for player $i$. (4) and (5) are the condition for the existence of the sanction for $k_{1}$.

The condition for sequential stability of the state $k$ includes one of (1) or (2) for every $k_{1} \in S_{i}^{+}(k, \pi)$ and (3)-(5) for every $k_{1} \in S_{i}^{*}(k, \pi)$.

3) The Condition for General Metarationality: In the case of GMR, the condition for the existence of sanction is not necessary. Therefore, we need to assume a very conservative player when we use the solution concept of GMR.
State $k_{l}$ in $S_{i}^{*}(k, \pi)$

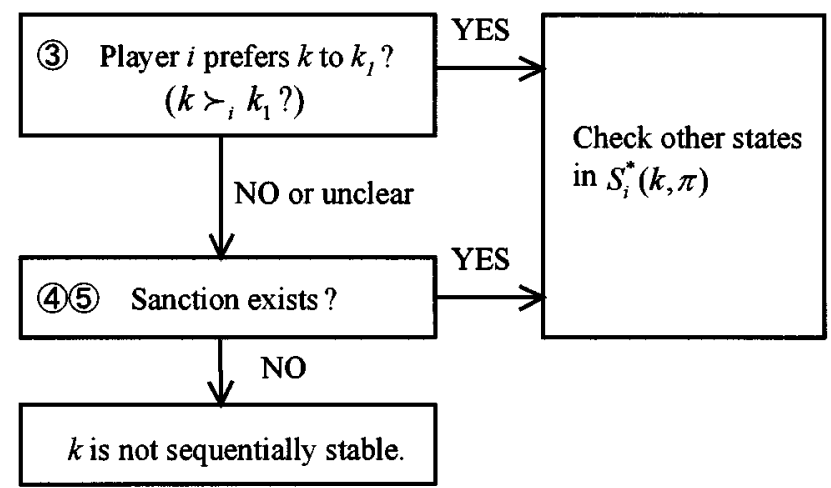

Fig. 4. Flowchart for specifying conditions for not moving to the state in $S_{i}^{*}(k, \pi)$ (sequential stability).

State $k_{l}$ in $S_{i}^{*}(k, \pi)$

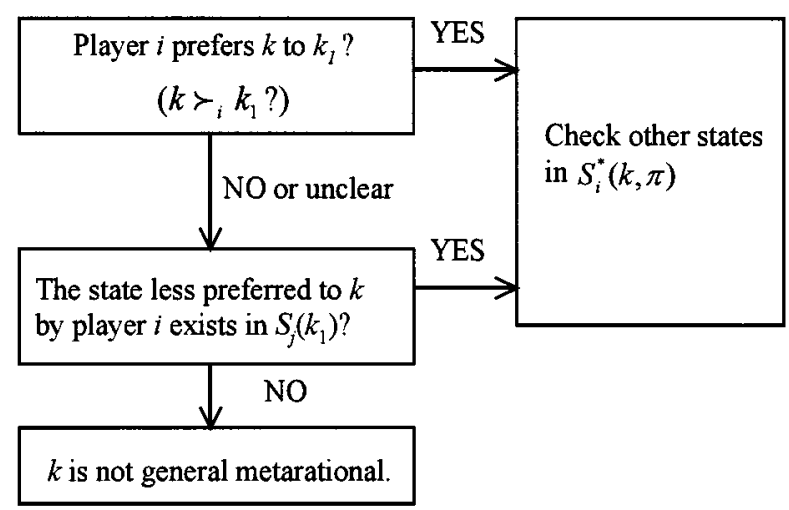

Fig. 5. Flowchart for specifying conditions for not moving to the state in $S_{i}^{*}(k, \pi)$ (general metarationality).

- For every $k_{1} \in S_{i}^{+}(k, \pi)$

$$
k \succeq_{i} k_{2} \quad \exists k_{2} \in S_{j}\left(k_{1}\right) .
$$

If $S_{j}\left(k_{1}\right) \cap \Phi_{i}^{-}(k, \pi) \neq\{\varnothing\}, k_{2}$ can always be found.

In case of the states in $S_{i}^{*}(k, \pi)$, the process for checking possibility of player $i$ s move is similar to the process for sequential stability. First, an analyst checks if player $i$ prefers $k$ to the state in $S_{i}^{*}(k, \pi)$. If an analyst obtains information that shows that player $i$ prefers $k$ to the state in $S_{i}^{*}(k, \pi)$, it needs to find the information showing existence of sanction (see Fig. 5).

- For every $k_{1} \in S_{i}^{*}(k, \pi)$,

$$
k \succ_{i} k_{1}
$$

or

$$
k \succeq_{i} k_{2} \quad \exists k_{2} \in S_{j}\left(k_{1}\right) .
$$

If $S_{j}\left(k_{1}\right) \cap \Phi_{i}^{-}(k, \pi) \neq\{\varnothing\}, k_{2}$ can always be found.

The sets of Nash stable states and sequentially stable state are the subsets of general metarational states [3]. Therefore, the conditions that the state is general metarational (14)-(16) are the necessary conditions for other solution concepts. That implies that the state which satisfies the conditions for Nash stability or sequential stability is more robust than the state which only satisfies the conditions for GMR. 


\section{Example of Robustness Analysis}

As an example, robustness analysis for the conflict shown in Fig. 6 is carried out. Since there are four states in the conflict, $|\Omega|=(4 !)^{2}=576$. Let us assume that an analyst's information set $\pi$ consists of the following eight pairs of preference orders:

$$
\begin{array}{ll}
A \succ_{1} B \succ_{1} C \succ_{1} D \text { and } B \succ_{2} C \succ_{2} A \succ_{2} D \\
A \succ_{1} B \succ_{1} C \succ_{1} D \text { and } C \succ_{2} A \succ_{2} B \succ_{2} D \\
A \succ_{1} C \succ_{1} B \succ_{1} D \text { and } B \succ_{2} C \succ_{2} A \succ_{2} D \\
A \succ_{1} C \succ_{1} B \succ_{1} D \text { and } C \succ_{2} A \succ_{2} B \succ_{2} D \\
C \succ_{1} A \succ_{1} B \succ_{1} D \text { and } B \succ_{2} C \succ_{2} A \succ_{2} D \\
C \succ_{1} A \succ_{1} B \succ_{1} D \text { and } C \succ_{2} A \succ_{2} B \succ_{2} D \\
C \succ_{1} A \succ_{1} D \succ_{1} B \text { and } B \succ_{2} C \succ_{2} A \succ_{2} D \\
C \succ_{1} A \succ_{1} D \succ_{1} B \text { and } C \succ_{2} A \succ_{2} B \succ_{2} D .
\end{array}
$$

Ordered sets and ordered reachable lists are shown as follows.

\section{Ordered Sets:}

$$
\begin{array}{ll}
\Phi_{1}^{+}(A, \pi)=\{\varnothing\}, \quad \Phi_{1}^{-}(A, \pi)=\{B, D\} \\
\Phi_{1}^{\wedge}(A, \pi)=\{\varnothing\}, \quad \Phi_{1}^{*}(A, \pi)=\{C\} \\
\Phi_{1}^{+}(B, \pi)=\{A\}, \quad \Phi_{1}^{-}(B, \pi)=\{\varnothing\} \\
\Phi_{1}^{\wedge}(B, \pi)=\{\varnothing\}, \quad \Phi_{1}^{*}(B, \pi)=\{C, D\} \\
\Phi_{1}^{+}(C, \pi)=\{\varnothing\}, \quad \Phi_{1}^{-}(C, \pi)=\{D\} \\
\Phi_{1}^{\wedge}(C, \pi)=\{\varnothing\}, \quad \Phi_{1}^{*}(C, \pi)=\{A, B\} \\
\Phi_{1}^{+}(D, \pi)=\{A, C\}, \quad \Phi_{1}^{-}(D, \pi)=\{\varnothing\} \\
\Phi_{1}^{\wedge}(D, \pi)=\{\varnothing\}, \quad \Phi_{1}^{*}(D, \pi)=\{B\} \\
\Phi_{2}^{+}(A, \pi)=\{C\}, \quad \Phi_{2}^{-}(A, \pi)=\{D\} \\
\Phi_{2}^{\wedge}(A, \pi)=\{\varnothing\}, \quad \Phi_{2}^{*}(A, \pi)=\{B\} \\
\Phi_{2}^{+}(B, \pi)=\{\varnothing\}, \quad \Phi_{2}^{-}(B, \pi)=\{D\} \\
\Phi_{2}^{\wedge}(B, \pi)=\{\varnothing\}, \quad \Phi_{2}^{*}(B, \pi)=\{A, C\} \\
\Phi_{2}^{+}(C, \pi)=\{\varnothing\}, \quad \Phi_{2}^{-}(C, \pi)=\{A, D\} \\
\Phi_{2}^{\wedge}(C, \pi)=\{\varnothing\}, \quad \Phi_{2}^{*}(C, \pi)=\{B\} \\
\Phi_{2}^{+}(D, \pi)=\left\{A, B, C^{\prime}\right\}, \quad \Phi_{2}^{-}(D, \pi)=\{\varnothing\} \\
\Phi_{2}^{\wedge}(D, \pi)=\{\varnothing\}, \quad \Phi_{2}^{*}(D, \pi)=\{\varnothing\} \\
S_{1}^{+}(A, \pi)=\{\varnothing\}, \quad S_{1}^{-}(\varnothing \pi)=\{\varnothing\}, \quad S_{1}^{\wedge}(A, \pi)=\{\varnothing\} \\
S_{1}^{*}(A, \pi)=\{C\} & S_{1}^{+}(B, \pi)=\{\varnothing\}, \quad S_{1}^{-}(B, \pi)=\{\varnothing\} \\
S_{1}^{\wedge}(B, \pi)=\{\varnothing\}, \quad S_{1}^{*}(B, \pi)=\{D\} \quad S_{1}^{+}(C, \pi)=\{\varnothing\} \\
S_{1}^{-}(C, \pi)=\{\varnothing\}, \quad S_{1}^{\wedge}(C, \pi)=\{\varnothing\}, \quad S_{1}^{*}(C, \pi)=\{A\} \\
S_{1}^{+}(D, \pi)=\{\varnothing\}, \quad S_{1}^{-}(D, \pi)=\{\varnothing\}, \quad S_{1}^{\wedge}(D, \pi)=\{\varnothing\} \\
S_{1}^{*}(D, \pi)=\{B\}, \quad S_{2}^{+}(A, \pi)=\{\varnothing\}, \quad S_{2}^{-}(A, \pi)=\{\varnothing\} \\
S_{2}^{\wedge}(A, \pi)=\{\varnothing\}, \quad S_{2}^{*}(A, \pi)=\{B\} \quad S_{2}^{+}(B, \pi)=\{\varnothing\} \\
S_{2}^{-}(B, \pi)=\{\varnothing\}, \quad S_{2}^{\wedge}(B, \pi)=\{\varnothing\}, \quad S_{2}^{*}(B, \pi)=\{A\} \\
S_{2}^{+}(C, \pi)=\{\varnothing\}, \quad S_{2}^{-}(C, \pi)=\{D\}, \quad S_{2}^{\wedge}(C, \pi)=\{\varnothing\} \\
S_{2}^{*}(C, \pi)=\{\varnothing\}, \quad S_{2}^{+}(D, \pi)=\{C\}, \quad S_{2}^{-}(D, \pi)=\{\varnothing\} \\
S_{2}^{\wedge}(D, \pi)=\{\varnothing\}, \quad S_{2}^{*}(D, \pi)=\{\varnothing\} . \\
\end{array}
$$

Let us think about the stability of the state B. Using the conditions shown in $\mathbf{C}$, the state $\mathbf{B}$ is stable if the following relationships are satisfied.

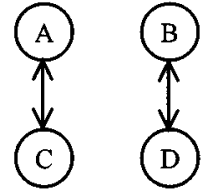

Player 1's feasible transition

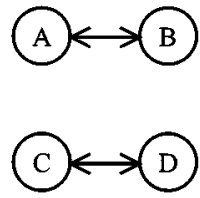

Player 2's feasible transition
Fig. 6. Conflict consisting of four states.

Nash Stability:

Player 1

$$
B \succ_{1} D
$$

Player 2

$$
B \succ_{2} A \text {. }
$$

Sequential Stability:

Player 1
(a)
$B \succ_{1} D$
(b)
$B \succeq_{1} C$

Player 2

$$
B \succ_{2} A
$$

$$
C \succ_{1} A \text { and } B \succeq_{2} C \text {. }
$$

\section{General Metarationality:}

Player 1 (a)

(b)

Player 2
(a)
(b)

$$
B \succ_{2} A
$$$$
B \succeq_{2} C \text {. }
$$

When two conditions [(a) and (b)] exist, the state B is stable if at least one of the two conditions is satisfied. The result of robustness analysis shows that limited number of preference relationships needs to be specified to guarantee the stability of a state.

\section{APPLICATION OF ROBUSTNESS ANALYSIS}

\section{A. Planning Conflict}

Environmental problem often involves conflicts between stakeholders. For example, Fang et al. [3] discuss the Garrison diversion unit conflict [5], which involves the governments of the United States and Canada, the government of Manitoba, environmentalists, etc. In the conflicts on environment, the alternative for mediating interests on development and environment is necessary. Therefore, it is important to guarantee the stability of a state.

In this section, the methodology of robustness analysis is applied to the conflict on hydropower generation and a river environment [6]-[8] (Fig. 7). There exist some reservoirs only for hydropower generation in a river basin. For the purpose of 


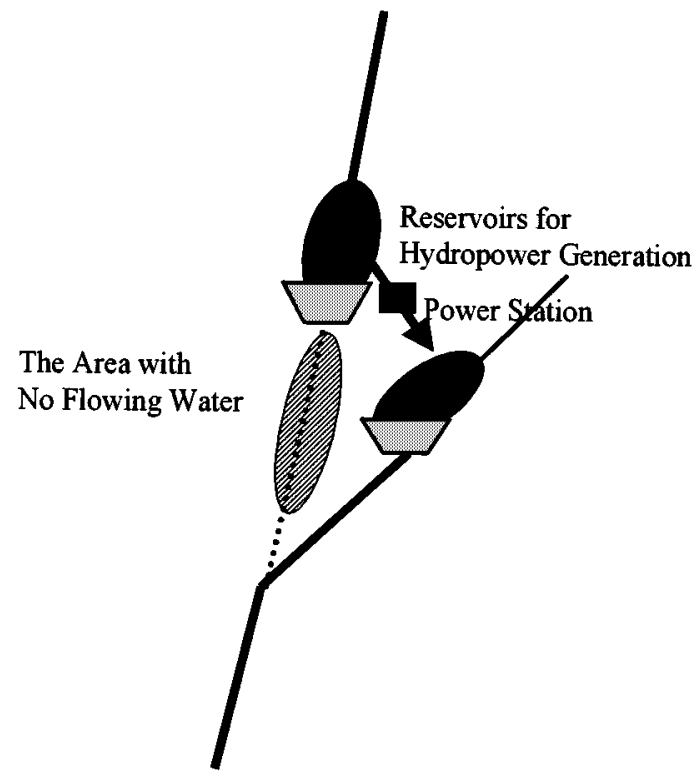

Fig. 7. Effects of hydropower generation on downstream river environment.

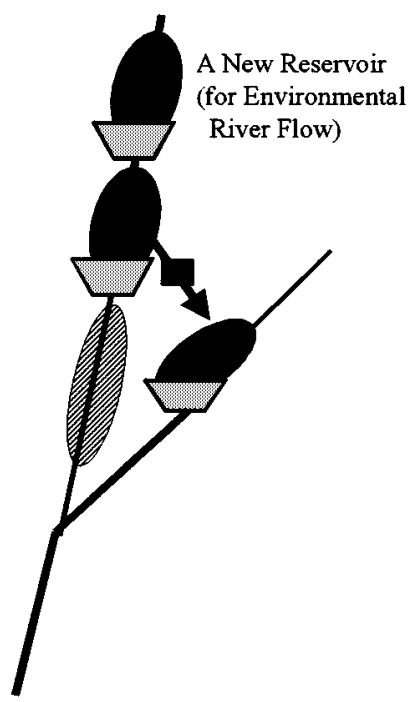

Fig. 8. Constructing a new reservoir for maintaining environmental river flow.

efficient use of potential energy, the water stored in reservoirs is often sent to the other reservoir directly. Bypassing water by pipelines may reduce a large amount of water downstream. That results in a shortage of river flow downstream. This shortage affects ecosystems, leisure, landscapes, sightseeing, and groundwater.

Several countermeasures (alternatives) to increase the flow of water are available. For example, local government can construct a new reservoir to obtain a storage capacity for environmental flow (see Fig. 8). In this case, local government can make a decision independently of existing user (hydropower generation). However, the resolution may result in an inefficient use of sites. Local government may not be able to find an appropriate site to construct an additional reservoir.

On the other hand, the power generation company may be able to reduce the level of hydropower generation (see Fig. 9). When this alternative is taken, the company has to generate elec-

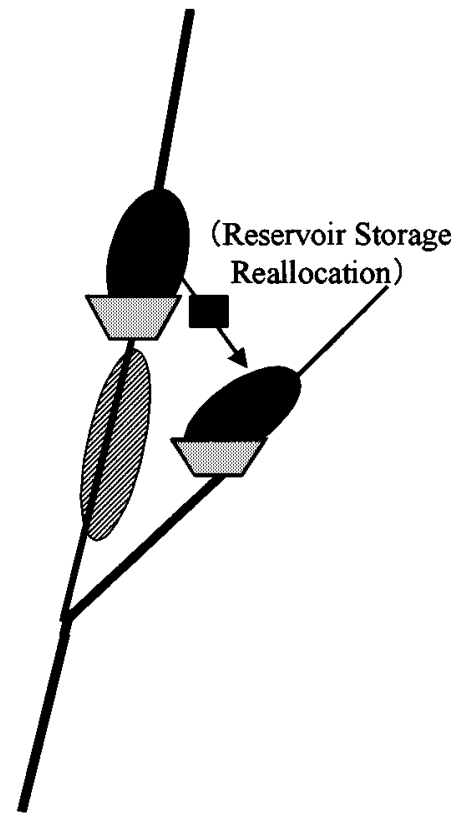

Fig. 9. Reservoir renewal (one example).

tricity by other means (other reservoirs, thermal power generation, etc.) If the scale of hydropower generation is reduced, the benefit to the power generation company is reduced. Therefore, the power generation company may ask the local government to compensate the loss. The transfer of benefit can be interpreted as net benefit reallocation of the project.

In this case, the power generation company represents the vested interests, while the local government represents the new interests of the river environment. A critical difference between them is that the power generation company (an existing user) can continue the hydropower generation in a status quo, while the local government cannot recover the river flow without taking any actions.

We assume two players. One is a local government and the other is a power generation company. In this conflict, the local government (Player 1) represents societal needs for better river environment. The purpose of the player is to conserve (or recover) the river environment by increasing discharge from a reservoir. We assume that the local government does not care about the benefit of a power generation company. The purpose of power generation company (Player 2) is to generate and sell electricity. Player 2 hopes to maintain the current level of hydropower generation. However, if it can achieve the cooperation with Player 1 (local government), it may have an incentive to change the current situation. Player 2's incentive depends on the net benefit which Player 2 can obtain.

Player 1 can maintain the status quo or construct a new reservoir by itself. These alternatives are called $S$ and $N$, respectively. Player 2 has the alternative that it stays in the status quo. The alternative is called $S$. Redevelopment solutions are not realized if at least one player takes these strategies.

We assume the case where several types of redevelopment solutions are assumed. Redevelopment solutions are represented by the combination of the alternatives on net benefit allocation and structural measures. This is assumed as follows. 
1) Player 1 can select the alternatives on net benefit allocation.

2) Player 2 can select the alternatives on structural measures. Player 1's alternatives for redevelopment solutions $\left(R_{k}\right)$ :

- Low payment level $\left(R_{L}\right)$.

- Medium payment level $\left(R_{M}\right)$.

- High payment level $\left(R_{H}\right)$.

Player 2's alternatives for redevelopment solutions $\left(A_{l}\right)$ :

- Using other power generation means $\left(A_{O}\right)$.

- Adding hydropower generation in another reservoir $\left(A_{D}\right)$.

- Upgrading the dam $\left(A_{U}\right)$.

Redevelopment solutions are represented by $R_{k} A_{l}$. The redevelopment solution $R_{k} A_{l}$ indicates that Player 1 takes strategy $R_{k}$ on net benefit allocation and Player 2 takes strategy $A_{l}$ on structural measure.

In order to realize redevelopment solutions, both players have to take the strategies compensating the loss (Player 1) and changing the status quo (Player 2), respectively. However, if Player 1 gives up compensating and preferred noncooperative solution $(N S)$, the state is moved from a cooperative solution $\left(R_{k} A_{l}\right)$ to a noncooperative solution $(N S)$. Similarly, if Player 2 gives up taking a structural measure for redevelopment, the state is moved from $R_{i} A_{j}$ to $N S$. On the other hand, the transition from $N S$ to $R_{k} A_{l}$ could not happen unilaterally. All of this shows that each player can cause the collapse of a cooperative solution unilaterally and the transition is an irreversible move.

Figs. 10 and 11 show $D_{1}$ and $D_{2}$ in the graph model for the planning conflict. The number of the feasible states is 20 . The collapse of cooperation is represented by irreversible and common moves.

\section{B. Information Structure in Planning Conflict}

In this planning conflict, we assume the following information structure:

$$
\begin{gathered}
R_{m} S \in\left\{\Phi_{1}^{-}\left(R_{k} A_{l}, \pi\right) \cap \Phi_{1}^{-}(N S, \pi) \cap \Phi_{1}^{-}(S S, \pi)\right\} \\
(m=O, D, U \quad k=O . D, U \quad l=L, M, H) \\
N A_{n} \in\left\{\Phi_{1}^{-}\left(R_{k} A_{l}, \pi\right) \cap \Phi_{1}^{-}(N S, \pi) \cap \Phi_{1}^{-}(S S, \pi)\right\} \\
(n=L, M, H \quad k=O . D, U \quad l=L, M, H) \\
S A_{n} \in\left\{\Phi_{1}^{-}\left(R_{k} A_{l}, \pi\right) \cap \Phi_{1}^{-}(N S, \pi) \cap \Phi_{1}^{-}(S S, \pi)\right\} \\
(n=L, M, H \quad k=O . D, U \quad l=L, M, H) \\
R_{m} S \in\left\{\Phi_{2}^{-}\left(R_{k} A_{l}, \pi\right) \cap \Phi_{2}^{-}(N S, \pi) \cap \Phi_{2}^{-}(S S, \pi)\right\} \\
(m=O, D, U \quad k=O . D, U \quad l=L, M, H) \\
N A_{n} \in\left\{\Phi_{2}^{-}\left(R_{k} A_{l}, \pi\right) \cap \Phi_{2}^{-}(N S, \pi) \cap \Phi_{2}^{-}(S S, \pi)\right\} \\
(n=L, M, H \quad k=O . D, U \quad l=L, M, H) \\
S A_{n} \in\left\{\Phi_{2}^{-}\left(R_{k} A_{l}, \pi\right) \cap \Phi_{2}^{-}(N S, \pi) \cap \Phi_{2}^{-}(S S, \pi)\right\} \\
(n=L, M, H k=O . D, U l=L, M, H) .
\end{gathered}
$$

Equation (30) reveals that temporary states are not preferred to the solutions which could become final solutions. We call $R_{k} A_{l}, N S$, and $S S$ "real solutions." This information enables us to classify states into two groups.

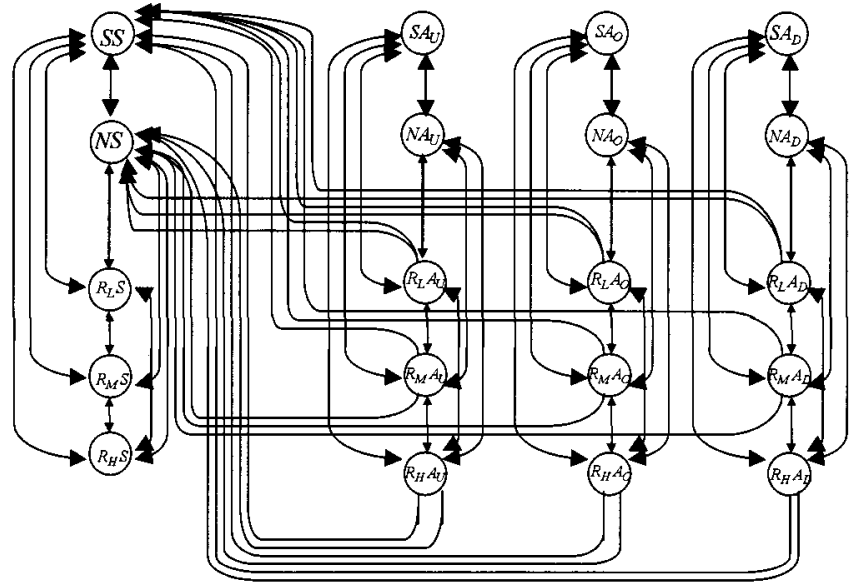

Fig. 10. Player 1's feasible transition between states.

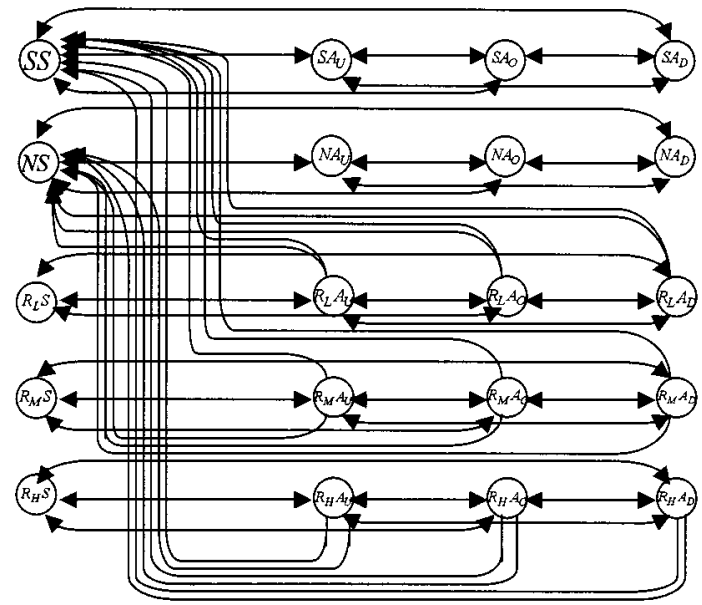

Fig. 11. Player 2's feasible transition between states.

On the other hand, it is also obvious that both players prefer the states in which its own payment is lower. That is

$$
\begin{gathered}
R_{L} A_{l} \succ_{1} R_{M} A_{l} \succ_{1} R_{H} A_{l} \\
R_{L} A_{l} \prec_{2} R_{M} A_{l} \prec_{2} R_{H} A_{l} \\
\left(A_{l} \text { means Player } 2\right. \text { s alternative). }
\end{gathered}
$$

Using this information, we can make the order between these states. Table I shows the ordered sets $\Phi_{i}^{+}\left(R_{M} A_{O}, \pi\right)$, $\Phi_{i}^{-}\left(R_{M} A_{O}, \pi\right)$, and $\Phi_{i}^{*}\left(R_{M} A_{O}, \pi\right)$ in the planning conflict.

\section{Players' Preferences and Social Efficiency}

As regards players' preferences, the following relationships exist.

- If $R_{k} A_{l} \succ_{1} N S$, Player 1 prefers the redevelopment solution $R_{k} A_{l}$ to constructing a new reservoir by themselves.

- If $R_{k} A_{l} \prec_{1} N S$, Player 1 prefers constructing a new reservoir by themselves to the redevelopment solution $R_{k} A_{l}$.

- If $R_{k} A_{l} \succ_{1} S S$, Player 1 prefers the redevelopment solution $R_{k} A_{l}$ to maintaining the status quo.

- If $R_{k} A_{l} \prec_{1} S S$, Player 1 prefers maintaining the status quo to the redevelopment solution $R_{k} A_{l}$. 
TABLE I

Player 1s Ordered Sets in the Planning Conflict $\left[\Phi_{i}^{+}\left(R_{M} A_{O}, \pi\right), \Phi_{i}^{*}\left(R_{M} A_{O}, \pi\right)\right.$, AND $\Phi_{i}^{-}\left(R_{M} A_{O}, \pi\right)$ ]

\begin{tabular}{c|c|c}
\hline$\Phi_{i}^{+}\left(R_{M} A_{O}, \pi\right)$ & $\Phi_{i}^{*}\left(R_{M} A_{O}, \pi\right)$ & $\Phi_{i}^{-}\left(R_{M} A_{O}, \pi\right)$ \\
\hline$R_{L} A_{O}$ & $R_{L} A_{U}, R_{M} A_{U}, R_{H} A_{U}$, & $R_{H} A_{O}, R_{L} S, R_{M} S, R_{H} S$, \\
& $R_{L} A_{D}, R_{M} A_{D}, R_{H} A_{D}$, & $N A_{O}, N A_{U}, N A_{D}$ \\
& $N S, S S$ & $S A_{O}, S A_{U}, S A_{D}$ \\
\hline
\end{tabular}

- If $R_{k} A_{l} \succ_{2} N S$, Player 2 prefers the redevelopment solution $R_{k} A_{l}$ to maintaining the status quo.

- If $R_{k} A_{l} \prec_{2} N S$, Player 2 prefers maintaining the status quo to the redevelopment solution $R_{k} A_{l}$.

On the other hand, redevelopment solution $R_{k} A_{l}$ is better than the noncooperative solution for the community consisting of both players if the following conditions (32) are satisfied:

$$
\begin{array}{ll}
R_{k} A_{l} \succ_{1} N S, & R_{k} A_{l} \succ_{2} N S \\
R_{k} A_{l} \succ_{1} S S, & R_{k} A_{l} \succ_{2} S S .
\end{array}
$$

If (32) is satisfied, redevelopment option $R_{k} A_{l}$ should be implemented from the viewpoint of a community, because $N S$ and $S S$ are Pareto-dominated by $R_{k} A_{l}$. However, in a planning conflict, if the profit obtained by a player can be improved by moving from $R_{k} A_{l}$ to another states, the player does not have an incentive to stay at $R_{k} A_{l}$.

\section{Application Results}

Table II shows the conditions that state $R_{M} A_{O}$ (Player 1 pays the cost at medium level and uses other power generation means) is Nash stable or sequentially stable for Player 1 (A) and Player 2 (B). The inequalities in Table II are the condition sets which the planning authority has to detect to confirm that $R_{M} A_{O}$ is a stable renewal alternative.

From $R_{M} A_{O}$, Player 1 can always move to low payment level. In other words, it is common knowledge that Player 1 has a UI from $R_{M} A_{O}$ to $R_{L} A_{O}$. Therefore, it is obvious that $R_{M} A_{O}$ is not Nash stable for Player 1 .

From Table II, we can obtain the following properties.

1) $\quad R_{M} A_{O}$ is stable for Player 1 if a) Player 1 prefers $R_{M} A_{O}$ to $N S$ and $S S$, and b) Player 2 can improve its payoff from $R_{L} A_{O}$ by changing its option from $A_{O}$ (using other power generation means) to $A_{D}$ (adding hydropower generation in other reservoir) or $A_{U}$ (upgrading the dam) and Player 1 prefers $R_{M} A_{O}$ to the resulting state $\left(R_{L} A_{D}\right.$ or $\left.R_{L} A_{U}\right)$ (b-1) or Player 2 prefers $N S$ or $S S$ to $R_{L} A_{O}$ (b-2).

If $R_{M} A_{O}$ is stable for Player $1,\{(\mathrm{a})$ and (b-1) $\}$ or $\{(\mathrm{a})$ and (b-2) $\}$ must be satisfied.

2) $\quad R_{M} A_{O}$ is stable for Player 2 if Player 2 prefers $R_{M} A_{O}$ to $N S$ or $S S$ (c), and Player 2 prefers $R_{M} A_{O}$ to $R_{M} A_{D}$ or $R_{M} A_{U}$ (d), Player 2 prefers $R_{M} A_{O}$ to $R_{L} A_{D}$ or $R_{L} A_{U}$ (e-1) or Player 1 prefers $N S$ or $S S$ to $R_{M} A_{D}$ or $R_{M} A_{U}$ (e-2).

If $R_{M} A_{O}$ is stable for Player 2, $\{(\mathrm{c})$ and (d) $\},\{(\mathrm{c})$ and (e-1) $\}$, or $\{(\mathrm{c})$ and (e-2) $\}$ must be satisfied.

Sequential stability needs player's ability to forecast counterpart's sanction. In order to lead players to the stable state, the planning authority needs not only to inspect players' preferences, but also to hold the information jointly with players. That is, specified preference order should be made common knowledge.

\section{E. Case With More Information}

Now we assume another situation where the planning authority can obtain additional information on players' preference. In this case, the number of conditions included in sufficient condition sets can be reduced. Table III shows sufficient condition sets for $R_{M} A_{O}$ s stability with following additional information.

1) Player 1 prefers arbitrary cooperative solution (reservoir renewal) $R_{k} A_{l}$ to both the status quo $S S$ and the noncooperative solution $N S$

$$
\begin{aligned}
R_{k} A_{l} \succ_{1} S S \quad \text { and } \quad R_{k} A_{l} & \succ_{1} N S \\
(k & =L, M, H, l=O, U, D) .
\end{aligned}
$$

2) Player 2 always prefers the structural measure $A_{O}$ (using other power generation means) to $A_{D}$ (adding hydropower generation in another reservoir) and prefers $A_{U}$ (upgrading the dam) to $A_{O}$

$$
\begin{aligned}
R_{k} A_{O} \succ_{2} R_{k} A_{D} \text { and } \quad R_{k} A_{U} \succ_{2} R_{k} A_{O} \\
\quad(k=L, M, H) .
\end{aligned}
$$

In this case, $R_{M}$ is the second largest payment for Player 1 and $A_{O}$ is the second preferred alternative for Player 2. $R_{M} A_{O}$ can be regarded as the compromising alternative.

Table III shows that $R_{M} A_{O}$ s stability depends on the tradeoffs of both players' preference between structural alternatives and payment. That is, the critical conditions for stability are that both players prefer $R_{M} A_{O}$ to $R_{L} A_{U}$.

\section{CONCLUSION}

In this paper, we proposed the application of robustness analysis to the conflicts where the information on players' preferences is incomplete. Robustness analysis is generalized to apply the situation where preferences of both players are not known by the third party. Then, the methodology was applied to the planning conflict between two players representing hydropower generation and river environment.

If the third party has only incomplete information on preferences of stakeholders, it is necessary to use a different methodology for coordination, which is different from one where complete information is available. The robustness analysis can become a useful approach to detect the stable and better alternatives. 
TABLE II

(a) Results of Robustness Analysis (State $R_{M} A_{O}$ ). (b) CONDITION SETS FOR PLAYER 2S STABILITY

\begin{tabular}{l|ll}
\hline Condition Set 1 & $S S \prec_{1} R_{M} A_{O}$ & $N S \prec_{1} R_{M} A_{O}$ \\
& $R_{L} A_{D} \preceq_{1} R_{M} A_{O}$ & $R_{L} A_{O} \prec_{2} R_{L} A_{D}$ \\
\hline \multirow{2}{*}{ Condition Set 2 } & $S S \prec_{1} R_{M} A_{O}$ & $N S \prec_{1} R_{M} A_{O}$ \\
& $R_{L} A_{U} \preceq_{1} R_{M} A_{O}$ & $R_{L} A_{O} \prec_{2} R_{L} A_{U}$ \\
\hline \multirow{2}{*}{ Condition Set 3 } & $S S \prec_{1} R_{M} A_{O}$ & $N S \prec_{1} R_{M} A_{O}$ \\
& $R_{L} A_{O} \prec_{2} S S$ & \\
\hline Condition Set 4 & $S S \prec_{1} R_{M} A_{O}$ & $N S \prec_{1} R_{M} A_{O}$ \\
& $R_{L} A_{O} \prec_{2} N S$ & \\
\hline
\end{tabular}

(a)

\begin{tabular}{|c|c|}
\hline \multirow{2}{*}{ Condition Set 1} & $S S \prec_{2} R_{M} A_{O}$ or $N S \prec_{2} R_{M} A_{O}$ \\
\hline & $R_{M} A_{D} \prec_{2} R_{M} A_{O} \quad R_{M} A_{U} \prec_{2} R_{M} A_{O}$ \\
\hline \multirow{2}{*}{ Condition Set 2} & $S S \prec_{2} R_{M} A_{O}$ or $N S \prec_{2} R_{M} A_{O}$ \\
\hline & $R_{L} A_{D} \preceq_{2} R_{M} A_{O} \quad R_{M} A_{U} \prec_{2} R_{M} A_{O}$ \\
\hline \multirow{2}{*}{ Condition Set 3} & $S S \prec_{2} R_{M} A_{O}$ or $N S \prec_{2} R_{M} A_{O}$ \\
\hline & $R_{M} A_{U} \prec_{2} R_{M} A_{O}$ \\
\hline \multirow[t]{2}{*}{ Condition Set 4} & $S S \prec_{2} R_{M} A_{O}$ or $N S \prec_{2} R_{M} A_{O}$ \\
\hline & $R_{M} A_{D} \prec_{1} N S \quad R_{M} A_{U} \prec_{2} R_{M} A_{O}$ \\
\hline \multirow{2}{*}{ Condition Set 5} & $S S \prec_{2} R_{M} A_{O}$ or $N S \prec_{2} R_{M} A_{o}$ \\
\hline & $R_{M} A_{D} \prec_{2} R_{M} A_{O} \quad R_{M} A_{U} \prec_{1} S S$ \\
\hline \multirow{2}{*}{ Condition Set 6} & $S S \prec_{2} R_{M} A_{O}$ or $N S \prec_{2} R_{M} A_{O}$ \\
\hline & $R_{M} A_{D} \prec_{2} R_{M} A_{O} \quad R_{M} A_{U} \prec_{1} N S$ \\
\hline \multirow{2}{*}{ Condition Set 7} & $S S \prec_{2} R_{M} A_{O}$ or $N S \prec_{2} R_{M} A_{O}$ \\
\hline & $R_{M} A_{D} \prec_{2} R_{M} A_{O} \quad R_{L} A_{U} \preceq_{2} R_{M} A_{O}$ \\
\hline \multirow[t]{2}{*}{ Condition Set 8} & $S S \prec_{2} R_{M} A_{O}$ or $N S \prec_{2} R_{M} A_{O}$ \\
\hline & $R_{M} A_{D} \prec_{1} S S \quad R_{M} A_{U} \prec_{1} S S$ \\
\hline \multirow{2}{*}{ Condition Set 9} & $S S \prec_{2} R_{M} A_{O}$ or $N S \prec_{2} R_{M} A_{O}$ \\
\hline & $R_{M} A_{D} \prec_{1} N S \quad R_{M} A_{U} \prec_{1} N S$ \\
\hline \multirow{2}{*}{ Condition Set 10} & $S S \prec_{2} R_{M} A_{O}$ or $N S \prec_{2} R_{M} A_{O}$ \\
\hline & $R_{M} A_{D} \prec_{1} S S \quad R_{M} A_{U} \prec_{1} N S$ \\
\hline \multirow{2}{*}{ Condition Set 11} & $S S \prec_{2} R_{M} A_{O}$ or $N S \prec_{2} R_{M} A_{O}$ \\
\hline & $R_{M} A_{D} \prec_{1} N S \quad R_{M} A_{U} \prec_{1} S S$ \\
\hline \multirow{2}{*}{ Condition Set 12} & $S S \prec_{2} R_{M} A_{O}$ or $N S \prec_{2} R_{M} A_{O}$ \\
\hline & $R_{M} A_{D} \prec_{1} S S \quad R_{L} A_{U} \preceq_{2} R_{M} A_{O}$ \\
\hline \multirow{2}{*}{ Condition Set 13} & $S S \prec_{2} R_{M} A_{O}$ or $N S \prec_{2} R_{M} A_{O}$ \\
\hline & $R_{L} A_{D} \preceq_{2} R_{M} A_{O} \quad R_{M} A_{U} \prec_{1} S S$ \\
\hline \multirow{2}{*}{ Condition Set 14} & $S S \prec_{2} R_{M} A_{O}$ or $N S \prec_{2} R_{M} A_{O}$ \\
\hline & $R_{M} A_{D} \prec_{1} N S \quad R_{L} A_{U} \preceq_{2} R_{M} A_{O}$ \\
\hline \multirow{2}{*}{ Condition Set 15} & $S S \prec_{2} R_{M} A_{O}$ or $N S \prec_{2} R_{M} A_{O}$ \\
\hline & $R_{L} A_{D} \preceq_{2} R_{M} A_{O} \quad R_{M} A_{U} \prec_{1} N S$ \\
\hline Condition Set 16 & $\begin{array}{ccc}S S \prec_{2} R_{M} A_{O} & \text { or } & N S \prec_{2} R_{M} A_{O} \\
R_{L} A_{D} \preceq_{2} R_{M} A_{O} & R_{L} A_{U} \preceq_{2} R_{M} A_{O}\end{array}$ \\
\hline
\end{tabular}

TABLE III-A

(a) Results of Robustness Analysis (StATE $R_{M} A_{O}$ ) With AdDitional INFORMATION. (b) CONDITION SETS FOR PLAYER 1S STABILITY.

(b) SUFFiCIENT CONDITION SETS FOR PLAYER 2S STABILITY

\begin{tabular}{l|c}
\hline Condition Set 1 & $R_{L} A_{U} \preceq_{\mathrm{I}} R_{M} A_{O}$ \\
\hline Condition Set 2 & $R_{L} A_{O} \prec_{2} S S$ \\
\hline Condition Set 3 & $R_{L} A_{O} \prec_{2} N S$ \\
\hline
\end{tabular}

(a)

\begin{tabular}{c|c}
\hline Condition Set 1 & $S S \prec_{2} R_{M} A_{O}$ or $N S \prec_{2} R_{M} A_{O}$ \\
& $R_{L} A_{U} \preceq_{2} R_{M} A_{O}$ \\
\hline
\end{tabular}

(b)

\section{REFERENCES}

[1] N. M. Fraser and K. W. Hipel, Conflict Analysis-Models and Resolutions. Amsterdam, The Netherlands: North-Holland, 1984.

[2] N. Howard, Paradoxes of Rationality. Cambridge, MA: MIT Press, 1971.

[3] L. Fang, K. W. Hipel, and D. M. Kilgour, Interactive Decision Making-The Graph Model for Conflict Resolution. New York: Wiley-Interscience, 1993.

[4] N. Okada, K. Tanimoto, and M. Arazoe, "A metagame analysis of conflicts between urban development and disaster prevention-An approach by robustness analysis" (in Japanese), J. Infrastruct. Planning Manage., vol. 29, pp. 79-92, 1995.

[5] N. M. Fraser and K. W. Hipel, "Solving complex conflicts," IEEE Trans. Syst, Man, Cybern., vol. SMC-9, pp. 805-815, Dec. 1979.

[6] N. Okada and H. Sakakibara, "Modeling a cost/benefit allocation game in a basin-wide reservoir renewal as a part of water resources reallocation," in Proc. IEEE Int. Conf. Syst., Man, Cybern., 1997.

[7] H. Sakakibara, D. Nakase, and N. Okada, "Game theoretic approach to decision making process under uncertainty in water resources reallocation" (in Japanese), in Proc. Annu. Conf. Soc. Risk Analysis, Japan-Section, vol. 10, Nov. 1997, pp. 130-135.

[8] _ - "Coordination of new and existing needs in water allocation conflicts - A case of the hydropower generation and environmental interests conflict" (in Japanese), Proc. Infrastruct. Planning, vol. 20, pp. 147-150, Nov. 1997

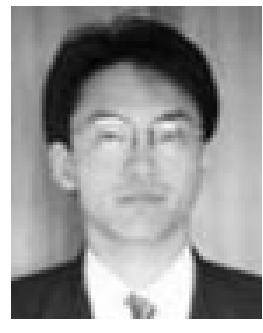

Hiroyuki Sakakibara (M'98) received the B.S. degree in civil engineering, the M.S. degree in applied system science, and the Ph.D. degree in civil engineering, all from Kyoto University, Kyoto, Japan, in 1995,1997 , and 2001, respectively.

$\mathrm{He}$ is currently an Assistant Professor in the Department of Civil Engineering, Yamaguchi University, Yamaguchi, Japan. His major research interests are application of game theory to management of infrastructure projects, evaluation of urban structure, and decision making in disaster risk management.

Dr. Sakakibara is a member of the Japan Society of Civil Engineers (JSCE), the Society of Risk Analysis, and the Japan Society of Hydrology and Water Resources. 


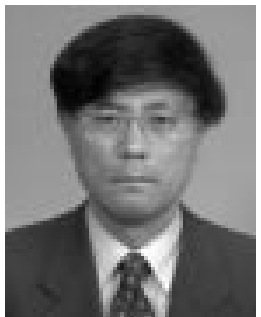

Norio Okada received the B.S. degree from the Faculty of Engineering, Kyoto University, Kyoto, Japan, in 1970, and the M.Eng. and Dr.Eng. degrees from Kyoto University in 1972 and 1977, respectively. $\mathrm{He}$ also received an "Honoris Causa" in engineering from the University of Waterloo, Waterloo, ON, Canada, 1995

He is currently Professor of disaster risk management at the Disaster Prevention Research Institute (DPRI), Kyoto University. His major research interests are game theory, disaster and environmental risk management, and regional planning.

Dr. Okada is a member of the Japan Society of Civil Engineers (JSCE), the Society of Risk Analysis, the Japan Society of Hydrology and Water Resources, among others. In 1995, he received the JSCE Research Award for his application of game theory to cost allocation in water resources development.

Daisuke Nakase received the B.S. and M.S. degrees in civil engineering from Kyoto University, Kyoto, Japan, in 1997 and 1999, respectively.

Since 1999, he has been with Obayashi Corporation, Osaka, Japan.

Mr. Nakase is a member of the Japan Society of Civil Engineers (JSCE). 